\title{
LAS SUPUESTAS INSCRIPCIONES PÚNICAS Y NEOPÚNICAS DE LAS ISLAS CANARIAS
}

\section{THE SUPPOSED PUNIC AND NEOUNIC INSCRIPTIONS FROM THE CANARY ISLANDS}

\author{
ANTONIO TEJERA GASPAR* \\ $\mathrm{M}^{\mathrm{a}}$ ANTONIA PERERA BETANCOR**
}

Resumen: Estudiamos en este trabajo una serie de inscripcio-
nes alfabetiformes encontradas en las islas canarias de Lanza-
rote y Fuerteventura, diferentes a la escritura líbico-bereber,
conocida desde el siglo XIX. Han sido interpretadas como es-
critura latina, pero también como signos alfabéticos púnicos
o neopúnicos.

Palabras clave: Inscripciones púnicas, neopúnicas, latinas y líbico-bereberes.

En estas pocas líneas plantearemos algunas cuestiones sobre uno de los hallazgos más interesantes de caracteres alfabéticos encontrados en las islas Canarias, en 1983, dados a conocer en esa fecha por sus descubridores, José de León Hernández¹, Roberto Hernández $^{2}$ y Ma Antonia Perera (1983). Se documentaba por vez primera en Lanzarote un nuevo tipo de escritura, que se parecía bien poco a la líbico-beréber, que desde el último tercio del siglo XIX se había localizado en la isla de El Hierro (fig. 1), de la que hoy contamos con un repertorio epigráfico en todo el Archipiélago, que conforma, sin duda, un patrimonio de un valor extraordinario $^{3}$.

\footnotetext{
* Universidad de La Laguna (Tenerife)

** Cabildo de Lanzarote.

1. De León, Hernández y Robayna (1988): 129-201; id. (1995): 455-535; de León y Perera (1996): 49-105; de Hernández (1990): 83-89.

2. Hernández y Perera (1983); id. (1987): 59-78; id. (1990); Hernández et alii (1987): 223-294; Hernández y Perera (s/f).

3. Springer (2001); Springer, Perera y Marrero (2001: 6-13).
}

\begin{abstract}
In this article we study one series of alphabetical inscriptions from the islands of Lanzarote and Fuerteventura (Canary Islands), different to the lybico-berber alphabet, known in these islands from the XIX century. There are different interpretations about these inscriptions, as latin letters, and also as punic or neopunics.
\end{abstract}

Keywords: Inscriptions punic, neopunic, latin and lybicoberber.

La aparición de aquella escritura contribuiría al descubrimiento posterior de un buen número de yacimientos en esta isla, y en la cercana de Fuerteventura, siendo las únicas por el momento, en las que se ha documentado este alfabeto. Existe, sin embargo, cierto parecido con algunos signos de La Gomera, grabados en el asa de un recipiente de madera, aunque por el momento no se han podido determinar con toda certeza. Si bien hoy resulta relativamente fácil adscribir cultural e históricamente la escritura líbico-beréber, tanto la del continente africano, como la de las islas Canarias, mucho más controvertida es, sin embargo, la valoración de estos otros caracteres alfabéticos, a los que se les denominó desde su descubrimiento como escritura "latina cursiva pompeyana", o de manera más simplificada "latina." Se trataba de signos alfabéticos en extremo diferentes a lo documentado hasta la fecha, por lo que se le buscó este otro origen, al observar ciertas similitudes con aquélla en los trazos de algunas letras. Esta denominación se ha ido generalizando, hasta el extremo 


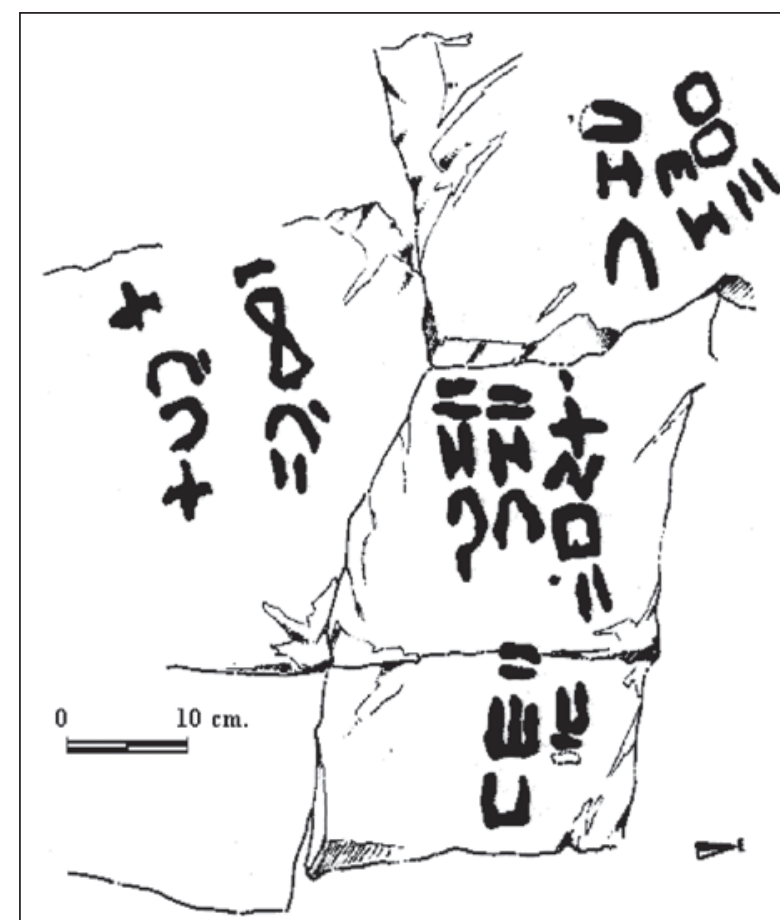

Figura 1. Inscripción líbica-bereber de la isla de Hierro

de que algunos investigadores, como veremos, los consideran propios de la citada escritura ${ }^{4}$.

Una dificultad similar sobre su denominación y adscripción cultural, se produjo en fechas cercanas, con el descubrimiento en el norte de África de un alfabeto en el que existía un cierto parecido con algunos signos de los encontrados en Canarias, y que al no poderlos definir tampoco de manera precisa, algunos investigadores, como Th. Monod (1993: 381-386) y M. Milburn (1983: 8-11), optaron por denominarlos con un término igualmente impreciso. El de "inscripciones enigmáticas" (fig. 4).

El problema de esta escritura radica no sólo en la determinación correcta de su contexto y adscripción cultural, sino en el de definir su denominación precisa. En un trabajo nuestro anterior, propusimos sustituir el término de "escritura latina" por otro menos comprometido, denominándola líbico-canaria, hasta tanto no pudiéramos precisar debidamente su adscripción cultural y cronológica ${ }^{5}$. Con este nombre compuesto queríamos expresar su origen norteafricano. Con el término

4. Para esta discusión se puede consultar el trabajo de Tejera y Perera (2011: 565-572).

5. Tejera y Perera (1996: 107-131); Tejera (1990: 533-542); Tejera y Chausa (1999: 69-74).

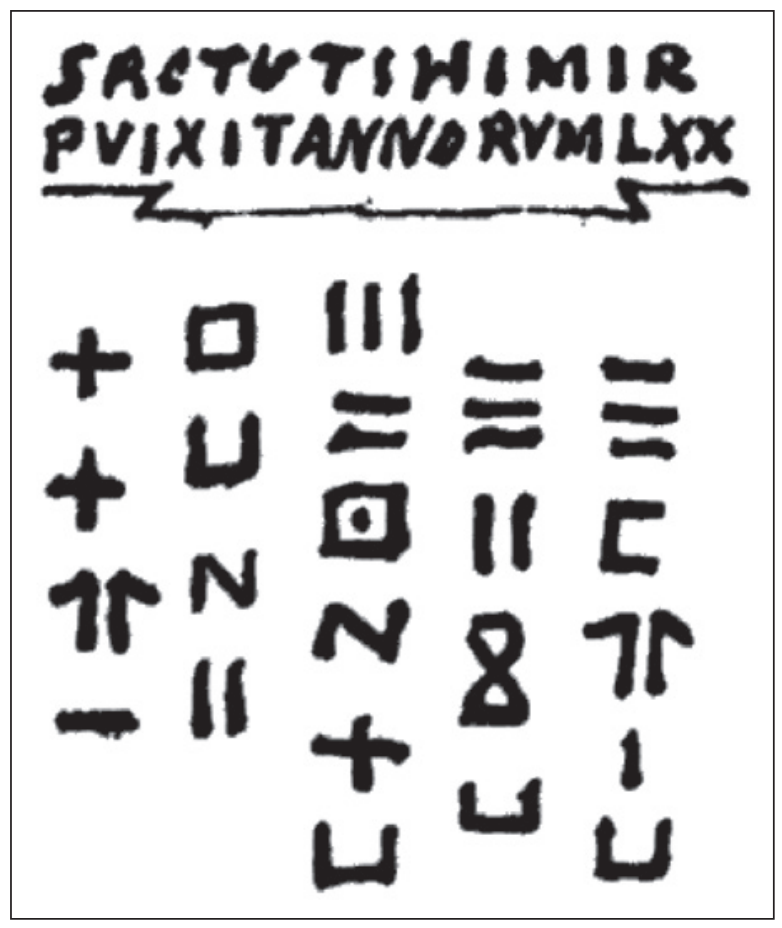

Figura 2. Inscripción bilingüe líbico-latina

libio se hacía referencia al nombre de Libia, utilizado por los griegos para denominar al África mediterránea, situada al oeste de Egipto, que conocemos en la actualidad con el apelativo árabe de Magreb. Y con el de canario se ponía de relieve la única zona fuera del continente, las islas Canarias, en donde también se había localizado esta escritura. Nos referimos al yacimiento de "Bu Njem" en la actual Libia, en donde existen algunos caracteres que poseen mucha semejanza con los canarios, y para los que René Rebuffat ${ }^{6}$ había utilizado la denominación de "líbico de Bu Njem", o de alfabeto "Bujenien". Y como quiera que no se han podido adscribir de manera segura a ningún contexto cultural, el citado investigador quería que con esa denominación no se prejuzgara la naturaleza histórico-cultural de los textos hallados en este yacimiento, del mismo modo que hemos propuesto con los de Lanzarote y Fuerteventura (fig. 5).

6. Rebuffat (1969: 189-212, pp. 189-195: rôle des fortresses sahariennes", pp. 195-212, fouilles de Bu Njem, avec plan du camp et des principia); $i d$. (1972: 319-339); $i d$. . (1974-75 : 165-187) ; $i d$. (1975: 495-505); id..(1982: 188-199); id. (1983 : 911-919) ("signification d'inscriptions de Menaa, Castellum Dimmidi, Lambèse et $\mathrm{Bu}$ Njem (entre 158 et 259 ap. J.-C.)". "Le sens 'autel du cierge' rapporte à un culte en rapport avec Flore et les Floralia (3 mai). 


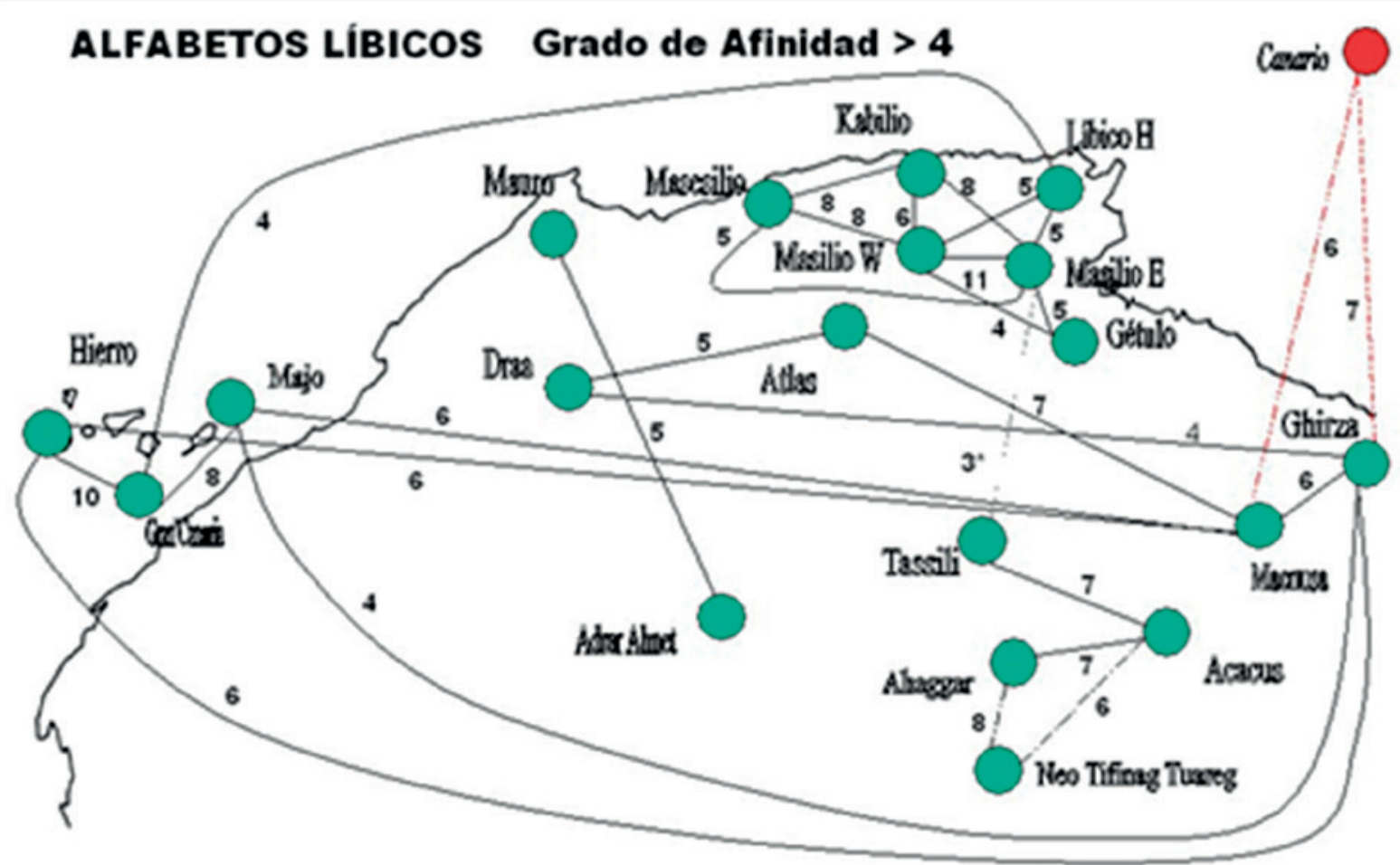

Figura 3. Mapa en el que figura la relación de los alfabetos líbico-bereberes norteafricanos y su afinidad con los de las islas Canarias, según J.A. Belmonte et alii (2001).

\section{HIPÓTESIS SOBRE SU ADSCRIPCIÓN CULTURAL}

La singularidad y novedad de este alfabeto ha planteado una seria discusión científica del máximo interés, sobre todo -como hemos señalado-, en lo que respecta a su adscripción cultural, propiciando una serie de hipótesis para explicar su origen y contexto cultural. De las diferentes propuestas -todas ellas muy discutidas, como veremos-, destacamos tres. Algunos investigadores han interpretado estos signos como propios del alfabeto latino; otros le han buscado un origen púnico o neopúnico, frente a quienes se han decantado por enmarcarlos en una tradición autóctona norteafricana, cuyo origen se podría remontar incluso a una etapa anterior a la presencia en el Magreb de las culturas protohistóricas mediterráneas. H. J. Ulbrich (1990: 7-319) los emparentó asimismo en sus primeras publicaciones con escrituras ibéricas, aunque más tarde se inclinaría por atribuirles igualmente una filiación latina (fig. 6).

La comparación con el alfabeto latino ha sido, sin duda, la hipótesis que ha hecho mayor fortuna, y aunque ciertamente parece existir una aparente afinidad de

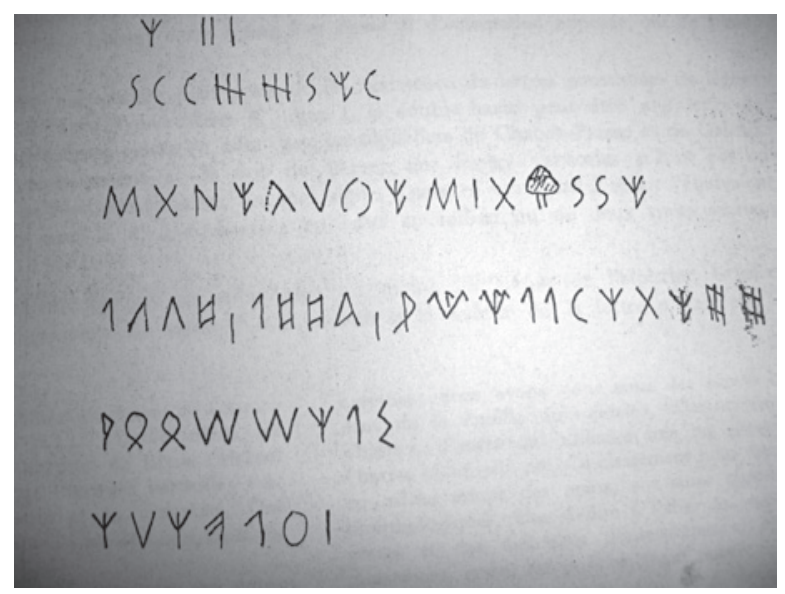

Figura 4. Escritura de Bu Njem en Libia, según R. Rebuffat

algunos caracteres con esa escritura, resulta, a nuestro juicio, muy difícil de adscribirlos en su totalidad como propios de aquél.

El supuesto origen latino de algunas inscripciones alfabéticas de las islas, ya había sido defendido por el investigador Pedro Hernández Benítez (1955: 182-186), 


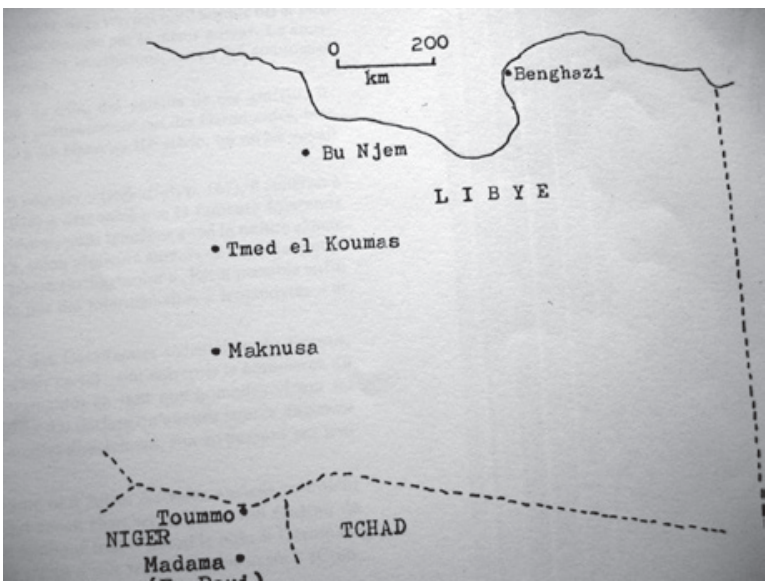

Figura 5. Ubicación del yacimiento de $\mathrm{Bu} \mathrm{Njem}$ en Libia, según R. Rebuffat.

quien en 1955 interpretó como tales unas líneas de texto halladas en Fuerteventura 7 . Se trata de unos epígrafes aparecidos en 1874 y 1878, en el barranco de la Torre, $\mathrm{y}$ en unas construcciones monumentales de trazado laberíntico halladas en Jandía. Fueron descubiertos por el Marqués de La Florida, don Luis Benítez de Lugo, y por el erudito majorero don Ramón Castañeyra (Álvarez Delgado 1964: 398-399), dados a conocer en la obra de Sabino Berthelot (1980: 220), quien los consideró "un fragmento de inscripción lapidaria con signos grabados muy parecidos a los de Los Letreros de la isla del Hierro" (ibid. 142). Se trata de un bloque pétreo del que ignoramos las medidas, que contenía cinco o siete signos dispuestos en posición vertical, aunque probablemente de lectura horizontal. J. Álvarez Delgado también consideró estos signos epigráficos como propios de aquélla escritura norteafricana, para la que propuso la siguiente lectura. Uno de ellos, lo lee (a) mdlrny y lo traduce por el tuareg "amadel-aranah", o amadaliranay "tierra mala"; y el otro, (b) idyn, lo considera un plural beréber de eidi con el significado de "perro" o bien como iudayan con el de "demonio" (Álvarez Delgado 1964: 399) (fig. 7).

El citado investigador P. Hernández Benítez (1955: 183), como decíamos, se había inclinado por considerarlas de origen latino-romana, para las que proponía una fecha próxima a nuestra era. Para defender su hipótesis utilizaba argumentos históricos, sosteniendo que las islas habían sido descubiertas por los romanos,

7. Un estudio clarificador y enjundioso sobre los supuestos caracteres latinos de Canarias puede verse en el trabajo de M. Ramírez Sánchez (2004: 2112-2130).

\begin{tabular}{|c|c|c|c|c|}
\hline Linea & Panel & Inscripción & & Transcripción \\
\hline 181 & M 2 & $M N \geqslant I V$ & & \\
\hline 182 & M3 & $N A I^{\prime} R N$ & & auaafran \\
\hline 183 & M 4 & 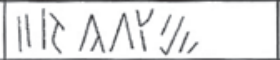 & $\mathrm{F}$ & \\
\hline 184 & M 5 & $|V| \wedge \mid$ & & ituiak / iaiacc \\
\hline 185 & M 6 & YASIWX & & tasi** \\
\hline 186 & M7 & AVNKIIAXMIDN & & aunt ${ }^{*}$ iagmidan \\
\hline 187 & M 8 & YASIMAY & & tasimat \\
\hline 188 & M9 & VAN'SAIIU & & uanisaen \\
\hline 189 & M 10 & VHY & & unt (uht) \\
\hline 190 & M 11 & $\| S W$ & & $\downarrow_{\text {mase }}$ \\
\hline 191 & M 11 & $N \lambda \| M$ & & $\downarrow$ unetan \\
\hline 192 & M 12 & $|\| i \wedge|$ & $\mathrm{F}$ & \\
\hline 193 & M 12 & N NXRIKI & $\mathrm{F}$ & $\mathrm{ag}^{\mathrm{a} * *_{\text {reki }}}$ \\
\hline 194 & M 13 & $\Lambda, \vee$ & $\mathrm{F}$ & \\
\hline 195 & M 13 & $\left|\lambda \wedge \backslash X_{i, 1}\right|$ & $\mathrm{F}$ & \\
\hline $196^{\circ}$ & M 14 & \|\|$\wedge \wedge$ & $\mathrm{F}$ & \\
\hline 197 & M 14 & $\|\wedge\| \lambda$ & $\mathrm{F}$ & \\
\hline 198 & M 15 & $\lambda^{Y}$ & $\mathrm{~F}$ & aut \\
\hline
\end{tabular}

Figura 6. Escritura líbico-canaria de Fuerteventura, que W. Pichler la ha considerado como alfabeto latino con su transcripción en castellano.

quienes más tarde “...dominaron a los naturales con los que comerciaban, visitando nuestro archipiélago periódicamente". Después de hacer una serie de disquisiciones históricas de la supuesta presencia de los romanos en las islas, especialmente en Gran Canaria, y en ésta de Fuerteventura, concluye diciendo que uno de los epígrafes, el localizado por el Marqués de La Florida en 1874, es un texto votivo para el que proponía la siguiente lectura: "Centum Vir Iulius Iovi Optimo Maximo", y "cuya versión a la lengua vernácula sería: ‘El Centuviro Julio a Júpiter Optimo Máximo" (ibid., 185), la misma que Álvarez Delgado había leído por el tuareg, "amadel-aranah". La otra inscripción encontrada cuatro años después, en 1878, por don Ramón Castañeyra, fue también considerada por Sabino Berthelot como signos de tipo líbico-beréber, aunque para nuestro investigador se trataba igualmente de un texto latino, similar al anterior, aunque en esta ocasión 


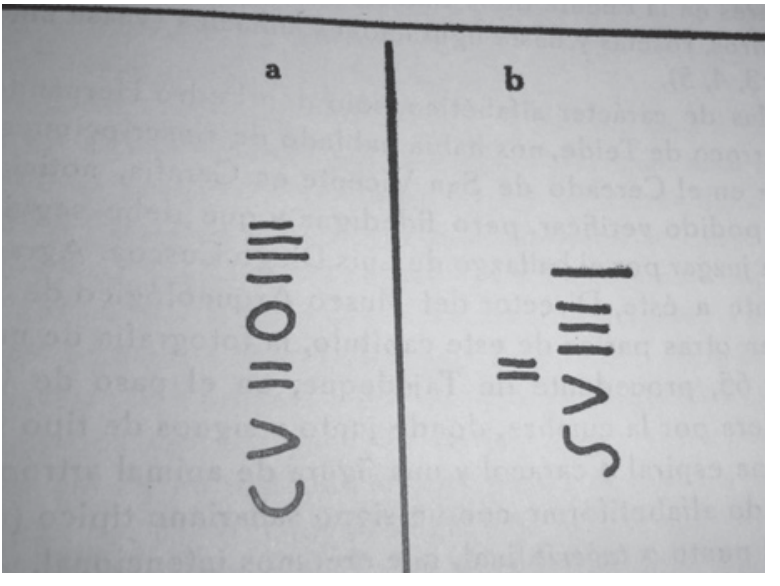

MDLNRY

IDYN

Figura 7. Escritura líbico-bereber de Fuerteventura, interpretada en otro momento como signos latinos, según J. Álvarez Delgado.

lo interpretaba sin discusión como una "piedra miliaria" que tradujo como "Piedra miliaria.-Cinco millas" (ibid., 185-186), leída, en cambio, por J. Álvarez como "iudayan".

\section{El origen púnico y neopúnico}

Otra de las explicaciones dadas a esta escritura ha sido la de considerarla de origen púnico o neopúnico. La raíz púnica de los signos la puso de manifiesto $R$. Muñoz, en su análisis de un conjunto de líneas de texto procedentes de Lanzarote y Fuerteventura. Este investigador pensaba que los caracteres considerados de escritura latina eran en realidad púnicos. Y partiendo de esta escritura establece una serie de lecturas de muchas de las líneas de texto de Fuerteventura, siguiendo el corpus publicado por W. Pichler (1992: 313-453; íd. 2003), así como también de las conocidas en Lanzarote. Con relación a una de las inscripciones de esta isla, quizá la más divulgada por ser de las primeras que se dieron a conocer -comúnmente leída por el latín como SINCICAVA (Balbín, Fernández y Tejera 1987: 19-54)-, este investigador piensa que "no es latina, sino púnica" (Muñoz 1993: 40).

Asimismo se refería R. Muñoz a la existencia de unos pocos textos bilingües líbico-púnicos de Fuerteventura, para los que propone su propia lectura $(\mathrm{Mu}-$ ñoz 1993: 34-35), y sobre los que quisiéramos hacer algunas consideraciones. El primer problema radica en saber cuál es el sentido de la lectura de las dos inscripciones para determinar si los caracteres considerados púnicos, son vocálicos o silábicos. El autor opta indistintamente por darle un sentido de lectura sinistrógira, es decir de izquierda a derecha, a los signos considerados púnicos, mientras que propone una lectura de abajo arriba para la escritura líbica. Pero como quiera que en estas escrituras la dirección puede ser diferente en cada caso, el problema no resulta fácil de solucionar. Más complejo aún es determinar si en realidad son púnicos estos signos escriturarios -los mismos, por cierto, que W. Pichler considera latinos-, ya que, a nuestro juicio, esta escritura nada tiene que ver con los alfabetos fenicio-púnicos que conocemos hasta el momento en la cuenca del Mediterráneo.

\section{La Piedra de Anaga}

La supuesta presencia de escrituras fenicio-púnicas en las islas, no es, como pudiera pensarse, un hecho nuevo, ya que a fines del siglo XIX se dio a conocer un breve texto con una serie de caracteres que se pretendieron emparentar, entre otros alfabetos, con el fenicio. Se hallaban inscritos en la conocida y muy discutida "Piedra de Anaga", encontrada el año 1886 en el entorno de Roque Bermejo (Anaga, Santa Cruz de Tenerife) (Mederos, Escribano y Ruiz 2000: 47), en la que Manuel de Ossuna $^{8}$, su descubridor, pretendió ver signos similares a los púnicos, hebreos, árabes e ibéricos, turdetanos y líbicos a la vez (Álvarez 1964: 398). Muchos investigadores consideraron este hallazgo como una piedra falsa, atribuyéndole "sus trazos a simples fisuras naturales del aragonito en que aparece" (ibídem) (fig. 8).

Se trata de una piedra cristalina de $8 \mathrm{cms}$ de longitud, que en una de las caras, convenientemente rebajada, se hallaban los citados signos de escritura. En recientes revisiones se ha vuelto a poner de manifiesto el valor de los caracteres inscritos en ella, considerándolos en esta ocasión de origen neopúnico", para los que se propone la siguiente lectura: "Debido a la fractura del sello, puede darse el caso de no estar completa parte de su lectura:]-ht II. Los dos últimos signos, dos trazos verticales paralelos consideramos que corresponden a un numeral, posiblemente el número dos. Si atendemos a la posibilidad de un antropónimo seguido

8. Ossuna y Van Den Heede (1889); Tarquis (1971: 169-177).

9. Mederos, Escribano y Ruiz (2000: 47). En el texto, los autores no se refieren a la escritura como de origen neopúnico, aunque sólo aparece tal clasificación en el pie de foto de la p. 47, en la que se dice: "Piedra de Anaga. Caliza cristalizada con sello inscrito neopúnico". 


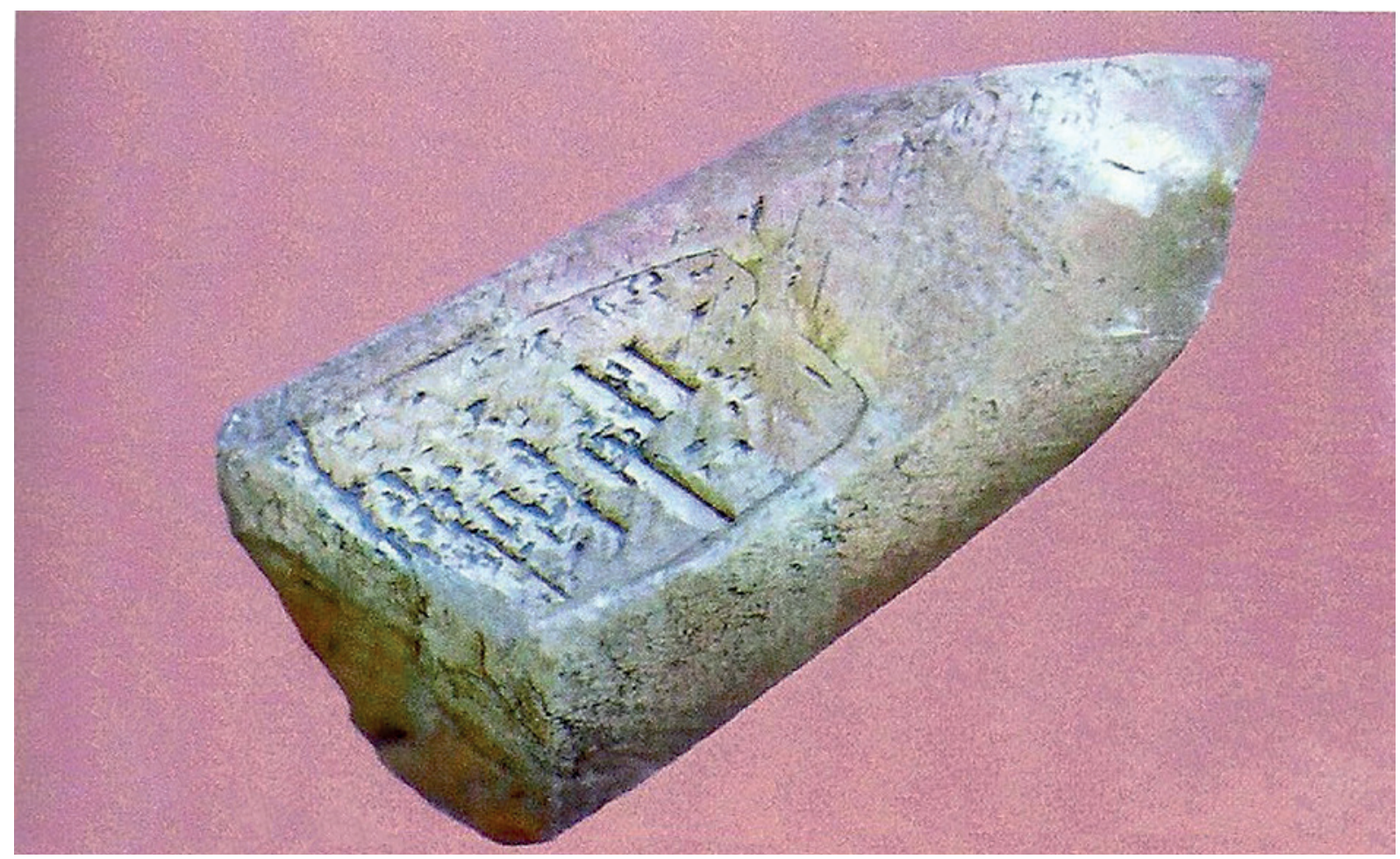

Figura 8. La denominada Piedra de Anaga cuyos grabados han sido interpretados como escritura púnica, según J. Farrujia.

de un numeral, podemos hallarnos ante un nombre finalizado en ' $h t$ "hermana", femenino de ' $h$ "hermano", con caída del primer 'aleph. Pero también hay otras alternativas a la utilización de un nombre personal, como puede ser la referencia a un producto, o también a una zona geográfica, origen probablemente de una determinada mercancía" (Mederos, Escribano y Ruiz 2000: 49). Por su parte, A. José Farrujia (2002) en un trabajo muy documentado sobre las circunstancias del hallazgo de este objeto, así como en todo lo relativo a su discusión científica, cree que "la presencia en ella de un nombre terminado en $h t$, "hermana" haría referencia a un teóforo o teónimo identificable con una deidad femenina, Tanit, paredra de Baal Hammón. En relación con este hecho, en la isla de Fuerteventura ya se ha señalado la presencia de una inscripción bilingüe, en púnico y líbico - sólo que en un soporte inmueble-, donde también se ha documentado el mismo teóforo" 10 .

Sobre el supuesto valor atribuido a los citados caracteres como propios de una escritura neopúnica, conviene tener en consideración dos aspectos. En primer lugar, nos parece pertinente determinar lo que se entiende con este término. Bajo tal denominación se

10. En el texto alude a la obra de R. Muñoz (1994: 35-36). agrupan todas las manifestaciones culturales arraigadas en la tradición fenicio-púnica, que después del 146 a.C. pervivieron en el Magreb bajo la ocupación romana (Fernández Ardanaz 1994: 97-114). La escritura neopúnica, conocida en distintos ámbitos del Mediterráneo, sigue la pauta de los caracteres fenicios antiguos, aunque con algunas evoluciones formales, que se desarrollarán en distintos contextos culturales, ya sean de tradición autóctona, en según qué lugares del Mediterráneo, o de los fenicios que continuaron sus modos de vida durante siglos en las costas de este mar, o en distintas zonas del norte de África, en donde esta escritura convivió con otras, como la latina, a lo largo de varias centurias después de la ocupación romana del continente. Esta asociación es muy común en muchos otros ámbitos norteafricanos, sobre todo en Túnez, en donde, en algunos casos lo hace con la escritura líbico-beréber, como está muy bien documentado en muchas estelas bilingües púnico-latinas. $\mathrm{O}$ coexistiendo también con el latín en dedicatorias de carácter público, como la conocida de la scaenae frons del teatro romano de Leptis Magna en Libia, que se fecha en el año 1 ó 2 de la Era.

No existe pues una escritura diferenciada que podamos definir como neopúnica, distinta a la de tradición 


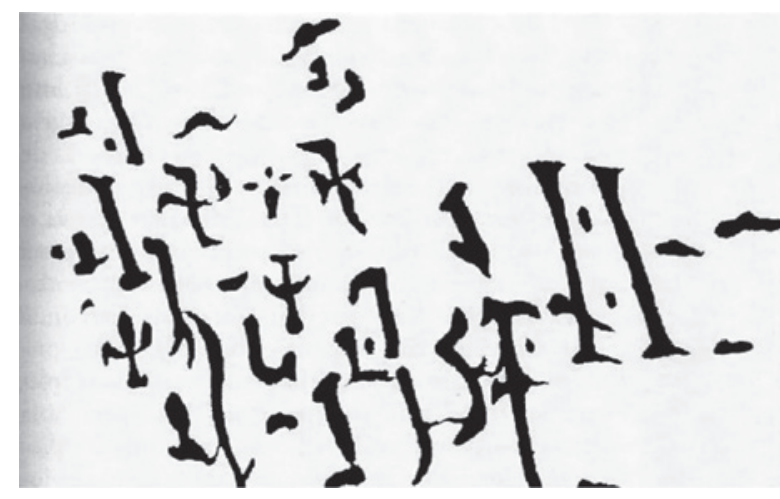

Figura 9. Supuestos signos de escritura púnica grabados en la Piedra de Anaga (Tenerife), según P. Tarquis.

fenicia, sino que se trata de los mismos signos de esta antigua escritura, cuya evolución gráfica se ha hecho a partir de otros más antiguos. Pero a la postre es el mismo sistema alfabético, y de grafía similar a aquélla, que se parece bien poco por cierto a la conocida como líbica o púnica de las dos islas citadas.

Pero sobre todo, para explicar un objeto de estas características con caracteres alfabéticos de escritura neopúnica, como la de la piedra de Anaga de Tenerife, o los textos púnicos, o los bilingües libio-púnicos señalados en Fuerteventura, habría que entenderlos, a nuestro juicio, con relación a la presencia de aquéllos en las islas, o en su caso, a unas posibles relaciones continuadas con el Archipiélago, ya que de lo contrario resulta muy difícil entender un objeto de estas características, completamente descontextualizado, en una isla en la que no existe ninguna evidencia arqueológica de esta cultura, ni nada hay tampoco, a nuestro juicio, "en la arqueología de las Islas Canarias que nos permita siquiera atisbar la presencia de gentes fenicio-púnicas, a pesar de que en época romana, este Archipiélago formó parte de las tierras extremas del mundo antiguo. Pero hasta donde sabemos por la investigación, bien contrastada en la actualidad, sólo alcanzamos a conocer que fueron pobladas por grupos libio-beréberes norteafricanos, en una fecha probable que iría desde fines del siglo I a.C., al primer tercio del s. I d.C. Y hasta tanto no existan evidencias materiales más precisas y mejor definidas sobre estas cuestiones, la supuesta presencia de fenicios y púnicos en estas islas, no deja de ser más que un problema histórico y arqueológico, reiteradamente estudiado desde antiguo, y que en ningún caso permite vincular las antiguas culturas canarias con ese mundo" (Tejera y Chávez, 2011:265).

Quisiéramos terminar estas consideraciones con las palabras autorizadas de M. Ramírez Sánchez "Ha transcurrido el tiempo suficiente para poder echar la vista atrás con la mirada reflexiva $\mathrm{y}$, sobre todo, crítica, que nos impone nuestra condición de historiadores. Pero a juzgar por varias publicaciones recientes, algunos colegas prefieren desempolvar estos trabajos como justificación de algunas líneas de investigación que parecen estar en boga hoy en día, a juzgar por el número de publicaciones que, de forma reiterada, pretenden demostrar, en ocasiones con más vehemencia que argumentos, la existencia de inscripciones neopúnicas y latinas en Canarias (González et alii 1995; González et alii 2003). Rescatada del olvido la piedra de Anaga nada parece contradecir la posibilidad de que los trabajos de Hernández Benítez puedan resucitar de la mano de algún historiador que, con vocación de epigrafista o filólogo, pretenda confirmar las teorías del sacerdote teldense" (Ramírez Sánchez 2004: 2120).

\section{La hipótesis de un origen africano: ¿es una escritura de los garamantes?}

Una última propuesta ha sido la de considerar que esta escritura, circunscrita por ahora en Canarias a estas dos islas orientales, y en el norte de África al área de la provincia de la Tripolitania, podría explicarse como la interpretación que hicieron las poblaciones libias de la escritura latina aprendida de los romanos, después de una primera etapa de contacto, y más tarde como resultado de un largo proceso de aculturación entre ambas comunidades. Se trata sólo de conjeturas, ya que nada de esto se halla bien definido por el momento, pero que cabría tenerla en consideración, en el supuesto de que el proceso de mestizaje se hubiera hecho con una cierta rapidez, ya que a pesar de que la destrucción de Cartago se fija en el 146 a.C., la presencia efectiva de Roma en esta parte del continente, sólo se manifiesta desde mediados del siglo I a.C., es decir, casi cien años después de aquélla primera incursión bélica. Y sobre todo, a partir del mandato de Augusto (27 a.C.-14 d.C.), reflexión a la que $\mathrm{W}$. Pichler se acerca también en su planteamiento.

Los signos escriturarios del yacimiento de " $\mathrm{Bu}$ Njem" están bien fechados en los siglos III y IV d.C. en una etapa perteneciente al Bajo Imperio. Tiempo que consideramos suficiente para que en el continente se hubiera producido ese proceso de mestizaje, mientras que para los signos de Canarias, por el contrario, no contamos con ninguna fecha, ni siquiera aproximada, para ubicarlos en una secuencia histórica determinada. Se ha hablado de que la presencia de esta escritura en 
las islas pudo ser resultado de la llegada de indígenas norteafricanos romanizados, de gentes aculturadas, que en el continente habían conocido, siquiera de manera rudimentaria, la lengua y la escritura latinas (Pichler 2003: 141-142). En ese caso, y siguiendo los modelos africanos de esos mismos procesos de aculturación, cabría pensar que tales acontecimientos hubieran tenido lugar al menos por las fechas a las que nos hemos referido para "Bu Njem", que por ahora -y con todas las reservas que requiere el caso-, pueden servir de referencia para explicar la presencia de estas gentes en Lanzarote y Fuerteventura, ya que en el caso canario, al no contar con datos cronológicos precisos, no podemos decantarnos por ninguna.

G. Camps, entre otros investigadores, ha planteado otra hipótesis no exenta tampoco de problemas, fundamentada en la posibilidad de que la base de este alfabeto se encontrara en la protohistoria norteafricana, pudiendo atribuírselo a los garamantes, o a otras etnias relacionadas con culturas mediterráneas de fines del II y principios del primer milenio a.C., y que se corresponde con una época muy anterior a la presencia romana, e incluso a la llegada de fenicios y griegos al Norte de África (Camps 1987: 202). En ese mismo sentido se manifestaba R. Rebuffat (1975: 167), al considerar que los garamantes, como otras gentes de la Tipolitania meridional, tuvieron en común una forma de escritura particular. Es en el Fezzan en donde se encuentran los signos más próximos a la escritura de " $B u$ Njem”, como asimismo en los yacimientos de "Maknusa" y en el "Tmed el-Koumas".

En apariencia podría pensarse que definir esta escritura como perteneciente a los garamantes sería como no decir nada, pero no es así, en absoluto. Por nuestra parte, creemos que es esta una perspectiva de investigación que encierra un gran interés por si estuviera relacionada con viejas escrituras prelatinas, aún por analizar, emparentadas de alguna manera con otras de la cuenca mediterránea, que acaso pudieran relacionarse con el sustrato cultural de los llamados "pueblos del mar". En este sentido conviene recordar aquí una vieja discusión, aún no resuelta, acerca de la presencia de carros de dos ruedas grabados en muchos yacimientos rupestres que se asociaron con la llamada "ruta de los carros saharianos", y que se creyeron vinculados con prototipos micénicos. Una revisión minuciosa de todos estos aspectos, en los que habrían de incluirse las que han sido consideradas "inscripciones enigmáticas", podría si no resolver el problema, al menos replantear estas cuestiones con una perspectiva diferente a la que manejamos actualmente.
Se trata una vez más de conjeturas, pero no cabría desdeñar que algo de eso pudiera estar reflejando algunos de los signos escriturarios que se encuentran en muchos lugares del Mediterráneo, entre los que existe un cierto aire de familia, aunque por el momento resulte muy arriesgado decantarse por una propuesta en este sentido, pero creemos que conviene dejar abierta esta otra posibilidad que complementaría algunas de las que aquí se hemos recogido.

Estas y otras muchas hipótesis creemos que pueden ser manejadas hasta tanto se conozca algo más sobre este aspecto relevante y novedoso de la epigrafía antigua de Canarias, y en especial de estos alfabetos, que por ahora sólo se han documentado en las islas de Lanzarote y Fuerteventura, y cuya determinación cultural ha de contribuir, sin duda, a entender mejor el origen antiguo de las etnias insulares.

\section{Escritura y poblamiento antiguo de las islas Canarias}

El poblamiento de Fuerteventura y Lanzarote puede servir de argumento para explicar el origen, y en parte la antigüedad misma de esta escritura. Resulta cuanto menos sugerente que nos encontremos ante un hecho de esta relevancia, cuál es la génesis de un alfabeto que sobre una base indígena preexistente, unas poblaciones asumieran y adoptaran algunos caracteres latinos hasta llegar a crear unas formas propias en donde se reflejaría la influencia de ambas tradiciones. De ser así, contaríamos con una documentación excepcional para fijar el poblamiento de estas dos islas, ya que en ese caso la llegada de sus gentes no se habría producido en una fecha anterior al 146 a.C., mediados del siglo II, período en el que, como hemos visto, los romanos penetraron en el Norte de África.

Nuestra propuesta es que el poblamiento de Canarias, y en concreto el de estas islas orientales, debió de haber tenido lugar a partir de la presencia romana en el Magreb, momento en el que las islas habrían sido pobladas con gentes castigadas por los romanos, a causa de las revueltas y de la tenaz resistencia que sostuvieron los beréberes ante el sometimiento de Roma, debido a una serie de acontecimientos que tuvieron lugar en los primeros años del siglo I d.C. Nos referimos a las insurrecciones de las etnias que habitaban un extenso territorio que se extiende desde el Atlas marroquí hasta Túnez, y que se alzarían contra el poder de Roma después de que les hubieran usurpado sus territorios. De todas ellas, destaca la protagonizada por la 
tribu de los Musulames que tuvo lugar entre los años 5 y 6 d.C. Con posterioridad, otras etnias formarían parte de la gran confederación que luchó de manera coaligada contra la presencia romana en el Magreb bajo el mando del célebre númida Tacfarinas, junto al que participaron muchas tribus en uno de los episodios más cruentos acaecidos durante el sojuzgamiento por Roma de las provincias africanas, y que coincidió con el mandato del emperador Tiberio (14-37), quien sofocaría estas revueltas iniciadas el 17 d.C., asesinando a Tacfarinas el año 22 d.C. Los enfrentamientos de las tribus beréberes no terminaron con su muerte, sino que continuaron durante varios siglos hasta el Bajo Imperio, con periodos muy cruentos, como ha sido bien estudiado por M. Rachet (1970) y M. Benabou (1976), entre otros historiadores (Tejera 2006: 96-98). Otros investigadores $^{11}$, sin embargo, sugieren la presencia de los romanos en el Archipiélago, pero relacionada con algún tipo de actividad comercial o exploratoria (Tejera y Chausa 1999: 69-74).

En todo caso, y hasta tanto no tengamos una secuencia cronológica y cultural más precisa, conviene dejar abiertas estas y otras posibilidades que el futuro permitirá determinarlas con más precisión.

\section{BIBLIOGRAFÍA}

ÁLVAREZ DELGADO, J. (1964): Inscripciones líbicas de Canarias. Ensayo de interpretación, La Laguna.

BALBÍN, R.; FERNÁNDEZ, M. y TEJERA, A. (1987): "Lanzarote Prehispánico. Notas para su estudio", XVIII Congreso Nacional de Arqueología: 19-54.

BELMONTE, J.A.; SPRINGER BUNK, R.; PERERA BETANCOR, M.A. y MARRERO, R. (2001): "Las escrituras líbico-bereberes de Canarias, el Magreb y el Sahara y su relación con el poblamiento del Archipiélago canario", Revista de Arqueología 245: 6-13.

BERTHELOT, S. (1980): Antigüedades Canarias. Goya Ediciones, Santa Cruz de Tenerife.

CAMPS, G. (1987): Les Berberes. Mémoire identité. Editions Errance, Paris ( $\left.2^{\mathrm{a} e d}\right)$.

FARRUJIA, A.J. (2002): El poblamiento humano de Canarias en la obra de Manuel de Ossuna y Van den Heede. Estudios Prehispánicos de Canarias 12. Gobierno de Canarias.

11. de León et al. (1988); Valencia y Oropesa (1990); Hernández y Perera (1991).
FERNÁNDEZ ARDANAZ, S. (1994): "La cuestión de la supervivencia del mundo púnico en el Mediterráneo Occidental de los siglos III-IV d.C", Coloquios de Cartagena, I. El Mundo púnico. Historia, Sociedad y Cultura (Cartagena, 17-19 de noviembre de 1990): 97-114. Murcia.

GONZÁLEZ ANTÓN, R.; DE BALBÍN BEHRMANN, R.; BUENO RAMÍREZ, P. y ARCO AGUILAR, Ma C. (1995): La Piedra Zanata. Santa Cruz de Tenerife.

HERNÁNDEZ BAUTISTA, R. (1987): "Los caracteres alfabéticos líbico bereberes del Archipiélago Canario", en M. Olmedo Jiménez (ed.), España y el Norte de Africa. Actas del I. Congreso HispanoAfricano de las Culturas Mediterráneas (Melilla 1984): 59-78. Granada.

- (1990): "Los grabados rupestres de Fuerteventura", en V. Valencia y T. Oropesa, Los grabados rupestres de Canarias. Viceconsejería de Cultura y Deportes, Gobierno de Canarias.

HERNÁNDEZ BAUTISTA, R. y PERERA BETANCOR, M.A. (1983): "Primeras inscripciones latinas en Canarias", periódico La Provincia.

HERNÁNDEZ BENÍTEZ, P. (1955): “Dos inscripciones latino-romanas", III Congreso Nacional de Arqueología: 182-186. Zaragoza.

HERNÁNDEZ CAMACHO, A.; CEJUDO BETANCORT, M.; ROBAYNA, M.A. et alii (1987): “Arqueología de la Villa de Teguise", I Jornadas de Historia de Fuerteventura y Lanzarote, T. II: 223-294.

HERNÁNDEZ DÍAZ, I. y PERERA BETANCOR, M.A. (s/f): Los grabados rupestres de la isla de Fuerteventura. Cabildo Insular de Fuerteventura.

HERNÁNDEZ, J. de (1990): "Los grabados rupestres de la isla de Lanzarote", en V. Valencia Afonso y T. Oropesa, Grabados Rupestres de Canarias: 83-89. Santa Cruz de Tenerife.

LEÓN HERNÁNDEZ, J. de; HERNÁNDEZ CURBELO, P. y ROBAYNA FERNÁNDEZ, M.A. (1988): "La importancia de las vías metodológias en la investigación de nuestro pasado, una aportación concreta: los primeros grabados latinos hallados en Canarias", Tebeto I: 129-201.

- (1995): "Los grabados rupestres de Lanzarote y Fuerteventura. Las inscripciones alfabéticas y su problemática", IV Jornadas de Estudios sobre Lanzarote y Fuerteventura: 455-535.

LEÓN HERNÁNDEZ, J. de y PERERA BETANCOR, M. A. (1996): "Las manifestaciones rupestres de Lanzarote", en Las manifestaciones rupestres de las 
Islas Canarias: 49-105. Dirección General de Patrimonio Histórico, Gobierno de Canarias.

MEDEROS, A.; ESCRIBANO COBO, G. y RUIZ CABRERO, L. (2000): "Pioneros. Manuel de Ossuna", Revista de Arqueología 236, Año XXI: 47.

MILBURN, M. (1983): "Enigmatic inscriptions at Bengazi (Cyrenaïca) and Er-Roui (N.E. Niger)", Club Newsletter 17: 8-11. London.

- (1984): "Sur quelques inscriptions énigmatiques des confines Nigéro-Fezzanais", Le Saharien 91 : 22-25.

- (1984): "Du Sud Fezzanais au Nord Nigérien à l'époque protohistorique", L'Universo LXIV, 5 : 130-149 et 197-199. Firenze.

- (1985): "Some unidentified inscriptions of Libya and Northern Níger", Discussions in Egyptology III: 31-37. Oxford.

- (1986) : "Le bilan actuel des inscriptions énigmatiques des confins Nigéro-Fezzanais", Le Saharien $98: 12-15$.

MONOD, TH. (1993): "Sur quelques inscriptiones sahariennes n'appartenant ni à l'ecriture arabe, ni à l'alphabet tifinagh", Memoire della Societá Italiana di Scienze Naturali e del Museo Civico di Storia Naturale di Milano, vol. XXVI, Fascicolo II: 381-386.

MUÑOZ, R. (1993): La piedra Zanata y el mundo mágico de los guanches. Santa Cruz de Tenerife, Museo Arqueológico.

OSSUNA Y VAN DEN HEEDE, M. de (1889): La inscripción de Anaga (Tenerife). Santa Cruz de Tenerife.

PICHLER, W. (1992): "Die Schrift der Ostinseln-Corpus des Inschriften auf Fuerteventura", Almogaren 23: 313-453.

- (2003): Las inscripciones rupestres de Fuerteventura. Cabildo de Fuerteventura.

RAMÍREZ SÁNCHEZ, M. (2004): "Saxa Sscripta, la búsqueda de inscripciones paleohispánicas y latinas en Canarias (1876-1955)", XV Coloquio de Historia Canario-americana: $2112-2130$.

REBUFFAT, R. (1969): "Deux ans de recherches dans le sud de la Tripolitaine", CRAI: 189-212.

- (1972): "Nouvelles recherches dans le sud de la Tripolitaine", CRAI: 319-339.

— (1974-75): "Graffiti en Libyque de Bu Njem (Notes et documents VII)", Libya Antiqua XI-XII: 165-187.
- (1975): "Trois nouvelles campagnes fouilles dans le sud de la Tripolitaine", CRAI: 495-505.

- (1982): "Recherches dans le désert occidental de Libye", CRAI : 188-199.

— (1983): “Ara cerei”, MEFRA 94, 2 : 911-919.

SPRINGER BUNK, R. (2001): Origen y uso de la escritura líbico-bereber en Canarias. Centro de la Cultura Popular Canaria.

TARQUIS RODRÍGUEZ, P. (1971): “Die Inschrift Von Anaga”, Almogaren II: 169-177.

TEJERA GASPAR, A. (1990): "Les inscriptions libyques-berbéres des îles Canaries", Convegno Internationale L'Arte e l'ambiente del Sahara preistorico: dati e interpretación: 533-542. Centro Studi Archeologia Africana, Milano.

TEJERA GASPAR, A. (2006): "Los libio-beréberes que poblaron las islas Canarias en la Antigüedad", en a. Tejera, Ma E. Chávez y M. Montesdeoca, $\mathrm{Ca}$ narias y el África Antigua: 96-98. Taller de Historia-41. Dirección General de Patrimonio Histórico, Cabildo de Fuerteventura y Centro de la Cultura Popular Canaria.

TEJERA GASPAR, A. y CHAUSA, A. (1999): "Les nouvelles inscriptions indigènes et les relations entre 1'Afrique et les îles Canaries", Bulletin Archéologique du Comité des Travaux Historiques C.T.H.S. nou. Sér. Afrique du Nord, fasc. $25: 69-74$, Paris.

TEJERA GASPAR, A. y CHÁVEZ, Mª E. (2011): "Fenicios y púnicos en las Islas Canarias. Un problema histórico y arqueológico". En Gadir y el Círculo del Estrecho revisados. Propuestas de la arqueología desde un enfoque social. Juan Carlos Domínguez Pérez (Ed. cient.), 2011. pp. 257-269. Editado por el Servicio de Publicaciones de la Universidad de Cádiz TEJERA GASPAR, A. y PERERA BETANCOR, $\mathrm{M}^{\mathrm{a}}$ A. (1996): "Las manifestaciones rupestres de Fuerteventura", en Las manifestaciones rupestres de las Islas Canarias: 107-131. Dirección General de Patrimonio Histórico, Gobierno de Canarias.

- (2011): "Las supuestas inscripciones latinas de Lanzarote y Fuerteventura", Sodalium munera. Homenaje a Francisco González Luis: 565-572. Ediciones Clásicas, Madrid.

ULBRICH, H.J. (1990): "Felsbildstationen auf Lanzarote", Almogaren XXI/2: 7-319. 